\title{
Advantages, challenges, and success factors in implementing information technology infrastructure library
}

\author{
Alice E. Cook, University of North Alabama, aecook@una.edu \\ Andrew S. Gann, University of North Alabama,agann2@una.edu \\ Daniel A. Ray, University of North Alabama,dray4@una.edu \\ Xihui Zhang, University of North Alabama,xzhang6@una.edu
}

\begin{abstract}
While Information Technology Infrastructure Library (ITIL) is best practice for Internet Technology Service Management (ITSM) and provides many advantages for an IT service provider, there can be many issues related to implementing this system. Many of these problems can be prevented when there is proper support. The purpose of this research paper is to provide a roadmap of best practices that smooth the adoption of ITIL. We first survey the advantages of ITIL adoption and implementation. We then provide a taxonomy of challenges that organizations often face when choosing to adopt ITIL as its ITSM framework. We determine critical success factors which, when implemented, will ameliorate those challenges. Lastly, we take a deeper look at the psychological components among stakeholders during ITIL adoption that lead to the identified challenges and provide insight into ways to overcome them.
\end{abstract}

Keywords: information technologies, information technology infrastructure library, ITIL, internet technology service management, ITSM

\section{Introduction}

Organizations who provide IT services must strive to achieve value for themselves while also providing value to their service consumers (Neničková, 2011). While there are many ways to manage a service, one of the most popular is Information Technology Infrastructure Library (ITIL) (Berntsen, 2017). ITIL organizes Internet Technology Service Management (ITSM) into a set of specialized organizational capabilities for enabling value to customers in the form of services (What is IT Service Management, n.d.). In order for value to be realized, the consumer must find utility when paying for the service provided. This places the burden of service management on the shoulders of the service provider. "ITIL offers a set of 'best practices' for managing IT services and is one of the most widely accepted approaches to IT service management in the world" (Chen \& Chou, 2010, p. 13).

In order to maximize value, IT service providers must have strong guidelines that define how they accomplish that goal (Neničková, 2011). The foundations of ITIL provide a list of the activities needed to implement the service value chain, guidelines for adapting and adopting ITIL principles, as well as best practices for technology service providers ranging from simple activities such as fulfilling a service request for new user access to problem management for recurring incidents. Using the Lean toolset, Obwegeser et al. (2019) developed a five-step process improvement framework (with guidelines and toolbox) for ITIL service operations. The five steps are: (1) Select: identify the most critical process; (2) Assess: assess the selected process; (3) Plan: plan process improvement initiatives; (4) Act: act on planned improvement initiatives; and (5) Evaluate: evaluate improvement efforts and plan further steps. Based on extant literature, 


\section{Issues in Information Systems}

Volume 22, Issue 2, pp. 187-198, 2021

Eikebrokk and Iden (2017) developed and empirically validated an ITIL Implementation Project Model. "The model includes significant antecedents, which provide a foundation upon which an effective ITIL implementation project can be built, as well as the pertinent effects of implementing ITIL" (Eikebrokk \& Iden, 2017). The antecedents include the following: senior management involvement, organizational commitment, group efficacy, project management capability, software quality, and organizational resources. The ITIL implementation progress (defined as the extent to which the IT function has implemented ITIL processes) includes the following: ITIL service strategy, ITIL service design, ITIL service transition, ITIL service operation, and ITIL continual service improvement.

The benefits of implementing ITIL beg the question - why don't all organizations adopt ITIL guiding principles and practices in their ITSM service delivery? While there are many advantages to adopting ITIL principles and adapting your service management to their best practices, there are also many barriers that stand in the way of an organization looking to increase value to their consumers through better service delivery via ITIL implementation. The purpose of this research paper is to provide a roadmap of best practices that smooth the adoption of ITIL. In this paper, we will look at the advantages of implementing ITIL, some of the challenges that can occur from implementation, and various methods and tools that can be used to conquer those challenges.

\section{Research background and method}

While there is currently ample research in this field, much of it is general, specific to international companies, or specific to technical fields. The literature does address specific barriers to ITIL adoption. Some of the most noted challenges are lack of commitment from upper management (Esteves \& Alves, 2013; Iden \& Eikebrokk, 2015), impact and efficacy of user engagement (Raflesia et al., 2017), and conflict in organizational cultures (Müller \& de Lichtenberg, 2018). Further, Chen and Chou (2010) compiled a list of barriers to ITIL success with the top five major challenges listed as culture shift, integration with current processes, ITIL/ITSM related knowledge, appropriate assessment tool, and clear measurement targets. However, the researched material lacked insight into the psychological barriers amongst organizational stakeholders (employees, management, etc.) that must be overcome for ITIL adoption success. When compiling a strategy to address common challenges to ITIL adoption, the psychological aspects of the stakeholders could prove to be just as germane to success as the organizational, cultural, and technical challenges.

We divided our research into three complementary categories. The goal of the first category was to examine current research into general advantages and challenges associated with implementation of ITIL in various business environments. To that end, we surveyed scholarly articles and academic papers dedicated to the subject. Our second area of research focused on specific issues and challenges faced in certain businesses that have attempted ITIL integration. We explored what went wrong with these attempts and what methods were used to mitigate the damage and successfully conclude the integration. The final phase of our research dealt with the psychological aspects related to ITIL integration. For this phase, we conducted research into techniques to counter psychological interference in business operations. This research included exploration of psychological journals, papers, and other resources.

\section{Results}

The results detailed in this section focus on the five areas of our research and are organized in the following order: a review of the organizational advantages to adopting and implementing ITIL, a survey of the types of general challenges faced during adoption and implementation, a survey of methods to counter these challenges, a review of the psychology of disruptive motivations among stakeholders during the 


\section{Issues in Information Systems}

Volume 22, Issue 2, pp. 187-198, 2021

implementation and change management phase, and finally best practices to overcome those psychological factors.

\section{ITIL adoption and implementation advantages}

Organizations worldwide recognize the worth of the ITIL framework. According to Berntsen (2017), "The most common reasons firms implement ITIL is to increase their operational efficiency and improve service quality or customer satisfaction" (p. 1). In addition, ITIL methodologies are associated with reduced IT costs. Further, the resultant better management of IT operational concerns, including compliance with business standards and requirements, directly and positively impacts certification and compliance with both local and international regulations (Chen \& Chou, 2010).

Kashanchi and Toland (2006) found that another significant advantage to adopting and implementing ITIL is that it can help align IT processes with business goals. In their investigation, they performed a literature review of Strategic Alignment, focusing on ITIL and various Strategic Alignment Models (SAM). They identified dominant alignment perspectives used to determine the extent to which ITIL adoption through SAM had a holistic effect on business. They found that ITIL adoption increased alignment by enhancing the communication between IT and business, by breaking down barriers and enabling the sharing of knowledge, and by increasing the ability of ITIL to support business strategies. Marrone and Kolbe (2011) subsequently found a statistically significant relation between mature ITIL implementation and high levels of IT/business alignment.

These advantages are mostly seen at the corporate level, but there are benefits that have been observed by lower-level employees as well. In Berntsen's (2017) study, she revealed that "[all] of the respondents reported that ITIL caused an increase in service quality. In addition, ITIL introduced stability to the workday, increased product quality and predictable services" (p. 32). According to Berntsen's research, the adoption of ITIL granted the participants of her study a more predictable and balanced working environment, and this, in turn, created more motivation and incentive for the employees.

Berntsen (2017) conducted in-depth research of Norwegian organizations who use ITIL in their business processes. The participants of her study included company employees who interacted with ITIL daily at their office in the areas of IT support and customer service. Most of Berntsen's study participants had over four years of experience working with ITIL, and the majority of them received ITIL training in Norway. Berntsen's results revealed that $67 \%$ of those studied felt that the organizational impact of the ITIL integration was positive, $72 \%$ of the participants noted ITIL implementation's positive impact on cooperation between employees, $83 \%$ suggested the use of ITIL provided greater awareness concerning how their work related to the work of others in the organization, 58\% noted an easier communication between employees and management, and $83 \%$ of participants identified an increase in collaboration between departments.

While some of these results are, arguably, subjective, objective indicators of the advantages of ITIL implementation are available as well. A document compiled by the Internal Revenue Service reported on how implementation of ITIL had affected some U.S. organizations.

For instance, Procter and Gamble saves $\$ 125$ million of IT cost, Shell Oil can upgrade their software in less than 72 hours and potentially save 6000 man-days working and 5 million dollars, Caterpillar raises their hit rate of target response time for resolving Web incidents from 30 percent to 90 percent of the time. (Chen \& Chou, 2010, p. 14) 


\section{Issues in Information Systems}

Volume 22, Issue 2, pp. 187-198, 2021

\section{General challenges faced in implementation and adoption of ITIL}

Even with the plethora of benefits imputed to the ITIL framework, organizations intent on ITIL implementation will inevitably face common challenges. The majority of organizations will encounter resistance to ITIL adoption from employees who oppose change, resist limitations being placed on their processes and procedures, differ with executive expectations, value ad-hoc creative solutions over proven methodologies, and worry about the complexity of the new processes (Chen \& Chou, 2010). Additionally, organizations which have not previously employed strict guidelines will undergo a cultural change. Employees accustomed to exercising localized decision making and control over their workflow may rankle at the dissipation of that control. Such resistance could lead to failure of the project.

ITIL promotes the need for measurement and report on the service quality, and it makes IT staff felt been [sic] watched constantly. In addition, adoption of ITIL represents great culture shift, [sic] the staff related to ITIL may fear their jobs [sic] become...obsolete, and this resistance will stall the process of implementation. (Chen \& Chou, 2010, p. 15)

A study performed by Esteves and Alves (2013) explored common elements of resistance to change when adopting ITIL. The research leveraged surveys and interviews collected during a case study implementation of the ITIL Configuration Management process at a Portuguese public organization (Esteves \& Alves, 2013). They concluded that the presentation of new technologies and solutions clashed with previously engrained procedures and habits. The challenge of resistance to change is exemplified by a common negative response among practitioners concerning the inconvenience of logging cases during periods of high workload (Berntsen, 2017). These actions required by managers, who also want incidents resolved quickly, led to a slow and bureaucratic process that was considered both demanding and time consuming to follow (Berntsen, 2017). With these findings in mind, resistance to change can be noted as a challenge commonly faced in the implementation and adoption of ITIL.

Another challenge faced when adopting and implementing ITIL is the successful engagement of users in adopting the updated procedures. Although ITIL enhances the delivery of IT services to customers, encourages the alignment of IT processes to business objectives, and supports effective incident management, ITIL does not provide a clear way to engage people outside IT who are involved (Yao \& Wang, 2010). When not all system users are engaged with an adopted system the quality of service is affected and incident handling becomes slow (Raflesia et al., 2017). Further, when users are faced with new procedures for handling their IT related incidents, use of the new processes can be sporadic and inconsistent. Bernsten (2017) found that customers would often short-circuit the new procedures by calling someone they knew in the IT department because they knew they could get their problem fixed straight away. A commonly shared difficulty for management was to create engagement and motivation among the wide variety of stakeholders who were needed to play a large role in the success of ITIL implementation and use (Pollard \& Cater-Steel, 2009). If management are not able to raise the necessary support and cooperation from the necessary stakeholders, the project will fail.

Beyond concerns related to the opposition of the workforce, organizations must also deal with technical issues they will inevitably encounter during the adoption of ITIL, such as integrating the new service delivery application, incorporating the new procedures into the existing procedures, developing accurate measurement tools, and training the workers in an effort to mitigate user error (Chen \& Chou, 2010).

\section{General factors contributing to successful ITIL implementation}

Our survey of related literature revealed a broad and varied set of factors contributing to successful ITIL adoption. For instance, Chen and Chou (2010) identify ten leading success factors: appointing a project 


\section{Issues in Information Systems}

Volume 22, Issue 2, pp. 187-198, 2021

manager, end user/customer support, ITIL training for IT staff, adaptability of IT staff to change, ITSM champions, sufficient allocations for IT staff, implementation team commitment to the project, effective change management, sufficient funding, and senior management commitment.

These factors generalize into four clear areas including: adequate support from executive management, proper involvement from and communication with middle managers, client buy-in and capability, and IT buy-in and training.

In other studies, the factors contributing to the success of ITIL adoption were much more focused but still fell into those four categories. Berntsen (2017) noted that "most of the respondents agreed that the success of ITIL depends on the degree of management involvement" (p. 35).

Likewise, another study exposed a very specific contributing factor to ITIL success.

Some resistance to adhering to the new documentation and communication process was experienced. ... A related challenge involved changing the focus from "crisis management" and "workarounds" to consideration of the real problems as defined by ITIL, and resolving the important underlying causes of incidents, measuring return on investment (ROI). (Pollard \& Cater-Steel, 2009, p. 173)

Pollard and Cater-Steel (2009) also noted that senior management support is the most important requirement for successful ITIL implementation. Like many other IT projects, having top management support ranks high as a contributor to successful ITIL implementation (Mohammadi et al., 2015).

Furthermore, a study by Iden and Eikebrokk (2015) focused on the impact of senior management involvement, organizational commitment, and group efficacy. It found that out of those three areas, group efficacy was shown to have the highest significance. "Group efficacy includes the level of competence, skills and methodological support that those involved in implementing the ITIL processes possess" (Iden \& Eikebrokk, 2015, p. 542).

Summarizing the top critical factors yields three common themes: Perceived level of top management support, group efficacy of the team involved in implementing ITIL, and maintaining a process-level focus throughout implementation.

\section{Psychological challenges affecting the implementation and adoption of ITIL methodologies}

Although the organizational and technical issues that arise from ITIL integration projects can play large roles in whether the endeavors are successful, we posit that an even more important factor is that of psychology. How stakeholders think and feel about changes such a project brings can have a greater influence on project outcomes than anything else. If the workers fully support the project and are excited and engaged, they will work hard to overcome problems that emerge from organizational and technical issues. Alternatively, if the employees do not welcome the project, no amount of skilled organization or technical acumen will suffice to counter their lack of participation.

Dent and Goldberg (1999) argue that it is not exactly that people resist change; what they really resist is loss, the loss of status, pay, or comfort. They assert that this opposition to loss is not the same thing as an opposition to change itself. While Dent and Goldberg's categories contain many of the psychological factors relevant to this discussion, there are two additional areas that should be added to help map back to our previous discussion about ITIL implementation resistance: lack of employee support and disruption of group dynamics. Introduction of change may trigger three types of concerns from employees, including 


\section{Issues in Information Systems}

Volume 22, Issue 2, pp. 187-198, 2021

irreconcilability concerns, ambiguity concerns, and contradiction concerns (Malhotra et al. 2021). In order to counter these challenges to successful ITIL implementation, we need to fully understand what causes such resistance.

The lines drawn between these categories are flexible, and some of the issues could bleed over into other categories, but for the sake of clarity, we have grouped these psychological factors into the following categories: worry about loss of status and/or pay, employee uncertainty, fear of failure, and disruption of group dynamics. In this section, we examine those issues more closely, and discuss various methods to address them in the next.

\section{Worry about loss of status and/or pay}

It is normal for stakeholders to feel a sense of loss when being forced to abandon comfortable and familiar processes for new and unfamiliar methods. One way this loss could manifest is through the worry about diminishing status and/or worry about a decrease in pay. There are two main causes for this concern. Zander (1950) argues that one of these causes is management putting unilateral pressure on workers to make changes instead of allowing them to contribute to the discussions and decisions regarding changes. Another underlying factor could be, according to Flower (1962), that management excludes employees from contributing to the specific way in which the change will be implemented and instead insists on exactly how everything will be accomplished. Both of these scenarios have the same result, demeaning the employees involved in the project and lowering their status from professionals in their fields to insignificant participants.

If employees feel under stress due to perceived threats associated with the project, they may respond in inappropriate or disruptive ways. It is often instinctual for others to respond in kind. Floger et al. (2013) call this phenomenon "emotional contagion" and define it as "a tendency for emotions to automatically mirror or mimic the emotional response to another, leading to a synchronization of emotional experience and reciprocation of behavioral responses to emotion" (p. 51). Managers need to be aware of this tendency and guard against it. Lashing out at employees who are emotionally vulnerable will only add to their stress and fear.

\section{Employee discomfort}

ITIL implementation is a massive undertaking that requires extensive training, a great deal of time, and copious amounts of additional work. Its implementation naturally upsets the normal flow of job activities for employees. Employees who have established a certain comfort level with their previous methods may find that an exceptional effort is required to establish a comfort level with the new processes. This could be even more daunting for workers who do not currently find their workload manageable. Flower (1962) adds to this concept by explaining that employees who currently feel overwhelmed by their workload will resist the thought of added work even more strongly.

\section{Lack of employee support}

Zander (1950) argues that a lack of employee support can come about when people who are affected sense overwhelming forces preventing them from changing. These forces could come in the form of insufficient time to learn the new processes, insufficient time to complete all required duties, insufficient support from management, insufficient participation from team members, etc. 


\section{Issues in Information Systems}

Volume 22, Issue 2, pp. 187-198, 2021

Additionally, Flower (1962) claims that workers will most likely resist change until they understand who will benefit from it, what the change will entail, why the change is necessary, when the change will be implemented and completed, and where the change will occur. Zander (1950) agrees and argues that the people who will be affected by the change must understand the nature of the change. He continues to explain that resistance will occur if the nature of the change is unclear and open to varying interpretations.

\section{Disruption of group dynamics}

A final psychological factor that can lead to ITIL implementation failure is that of a disruption of group dynamics. Departments and teams develop a social structure after having worked together for some time, a sort of rhythm that permeates their interactions. When this rhythm is disrupted, it can be difficult to recapture. Lawrence (1954) explains that often managers or staff specialists will be so focused on the technological aspects of the upcoming change that they will overlook the dynamics of the affected departments or teams. This oversight could lead to confusion, loss of status for some, uncertainty regarding who is expected to do what, etc.

\section{Solutions to psychological issues}

In order to adequately address psychological factors that can lead to implementation failure, it is vital that the underlying causes be identified. Incorrect categorization of these issues would lead to ineffectual remediations which address the symptoms instead of the disease. To address the three types of concerns (i.e., irreconcilability concerns, ambiguity concerns, and contradiction concerns) from the employee triggered by the introduction of change, Malhotra et al. (2021) suggested that we respond with the strategy of redirecting, reinforcing, and reassuring, respectively. As previously noted, our survey of the literature identified four areas: worry about loss of status and/or pay, employee discomfort, lack of employee support, and disruption of group dynamics. We address each in turn.

\section{Worry about loss of status and/or pay}

Flower (1962) stresses the importance of not imposing change on the workers, but instead empowering them to assist with molding the necessary change. He argues employees should be encouraged to participate in every step of the process, so they will develop an ownership of the project. Coch and French (1948) agreed and went even further to claim that participation in the change discussions was the primary way to defeat opposition to change. However, Lawrence (1954) argues that that perspective is too shallow. He claims that we must dig deeper into the causes beneath this resistance to discover a working solution. Lawrence claims that the group in Coch and French's study at Harwood that had the lowest results when it came to accepting change was motivated by more than just a lack of participation. He asserts that, instead, they were motivated by a loss of status. Lawrence claims that the fact that the group was not consulted or invited to participate had a greater effect than the simple fact that they did not participate. They saw the oversight as a disparagement of their abilities and skills. In order to counter that response, it is important that managers and staff specialists acknowledge that workers in the various affected areas are specialists in their fields and that they will have information to contribute. Managers and leaders should welcome their participation and engage them whenever a question arises that falls under their purview.

Additionally, managers can take steps to counter emotional contagion by focusing on keeping interactions positive and calm. Managers and supervisors need to "promote positive interactions, to encourage employees to engage in conversation that builds co-workers up, and therefore will overall improve positive communication in a business" (Mye, 2015, p. 13). Handling issues in a positive manner can go far toward countering fear of loss of status. 


\section{Issues in Information Systems}

Volume 22, Issue 2, pp. 187-198, 2021

\section{Employee discomfort}

Employee discomfort centers mainly around the amount of time that will be required in training and in dealing with increased workload (at least on a near-term basis). Flower (1962) offers two ways for managers to allay this difficulty. First, managers should establish communication with each individual whose job will be impacted by the ITIL implementation. They should discuss how these changes are affecting their workloads and other aspects of their jobs. They should listen to the employee's concerns and find ways to address them satisfactorily for both parties. Flower also suggests that managers should ensure that changes are made in a way that is nonthreatening. All employee concerns should be considered and discussed with the employee in a way that makes them feel heard. Managers need to make an effort to foster a sense of ownership and participation in the workers instead of acting as dictators and simply ordering them to cooperate.

\section{Lack of employee support}

Lack of employee support is rooted in two areas: employee uncertainty of success and employee confusion. Employee uncertainty of success stems from a sense of not having control over factors influencing outcomes. Flower (1962) addresses this concern and offers two methods to overcome it. First, picking the right time to implement the change can make a considerable difference relating to the ability to control outside factors. For those businesses that have busier seasons and slower seasons, the most intensive aspects of implementation should occur during times that are least busy. Secondly, Flower recommends setting aside time to train employees in problem-overcoming strategies. Discussions concerning possible conflicts and problems should be held regularly and should result in strategies with which all level of workers and managers are comfortable.

Managers can also foment employee support by removing the stigma that is attached to doubt and uncertainty. One way they can do this is to promote learning from mistakes. Williams (1998) calls these "smart mistakes" and defines them as mistakes that have negative consequences but that have been carefully examined and evaluated so that they may lead to more advantageous results in the future. By encouraging employees to think of failure as a learning experience that can lead to better things instead of merely a negative reflection of their worth, managers can take away some of the associated fear.

Finally, confusion amongst employees typically results from a lack of sufficient, clear, and comprehensive communication. Proper communication can reduce or even eliminate confusion and lead to more successful implementation projects. To avoid confusion related to important product information, Lawrence (1954) recommends that managers:

- Use terms that are enterprise-wide and understood by all participating parties.

- Continue working with employees until it is clear that all parties understand the information.

- Write new job descriptions that will clear up any misunderstandings about which employee is expected to fulfill which tasks.

- Facilitate communication among all related parties by emphasizing that as a role of the manager or by assigning someone specifically to that job.

\section{Disruption of group dynamics}

According to Flower (1962), managers and leaders must understand the role that each person plays in the group and what their individual responsibilities and perspectives are. Lawrence (1954) recommends that leaders work with employees to broaden their interests. It is obviously best to avoid a disruption of the 


\section{Issues in Information Systems}

Volume 22, Issue 2, pp. 187-198, 2021

social structure of the group; however, when such a disturbance cannot be prevented, helping the group members identify with other aspects of their department can help restore equilibrium.

\section{Discussion}

\section{Summary of content}

The literature review revealed advantages to ITIL adoption as well as challenges faced when an organization chooses ITIL as their ITSM framework of choice. The review also exposed clear factors that have a direct correlation to a successful ITIL implementation organizationally, culturally, technically, and, upon further analysis, psychologically. Until now, much of the literature focused on outward aspects of barriers and success factors, but there was a lack of focus on the psychological aspects, or internal factors, of the individual stakeholders and how those feelings contribute to the success or failure of the project. For the purpose of discussion, these barriers were grouped into categories and labeled, where appropriate at the user level, to provide insight into overcoming not only the organizational, cultural, and technical barriers, but psychological barriers as well.

Table 1 pairs not only the common barriers and challenges to successful ITIL adoption to their best-practice remedies, but also does the same for psychological barriers that were discovered in our research. The challenges of perceived loss, employee discomfort, lack of employee support, and disruption of group dynamics all have solutions to improve the psychological state of the end users during the transformation to ITIL.

Table 1: Categorized Factors for Successful ITIL Implementation

\begin{tabular}{|l|l|}
\hline \multicolumn{2}{|l|}{ General Challenges } \\
\hline Challenge/Barrier & Solution \\
\hline $\begin{array}{l}\text { Lack of senior } \\
\text { managerial support }\end{array}$ & $\begin{array}{l}\text { The project should not be related to any political decisions or political } \\
\text { interests in the company. }\end{array}$ \\
\cline { 2 - 2 } & $\begin{array}{l}\text { The project should be related to the corporate strategy as well as the IT } \\
\text { departmental strategy. }\end{array}$ \\
\cline { 2 - 2 } & $\begin{array}{l}\text { The project should relate high enough in the corporate structure to be } \\
\text { considered significant. }\end{array}$ \\
\hline Lack of funding & The project should be prioritized highly enough to warrant sufficient funding. \\
\hline Miscommunication & $\begin{array}{l}\text { Appoint a project manager to facilitate communication between management, } \\
\text { users, and employees. }\end{array}$ \\
\hline $\begin{array}{l}\text { The wrong } \\
\text { stakeholder delegates }\end{array}$ & Choose delegates from impacted departments as well as IT. \\
\cline { 2 - 2 } Low group efficacy & $\begin{array}{l}\text { Choose team members based on levels of competence, skills, and } \\
\text { methodological support. }\end{array}$ \\
\hline $\begin{array}{l}\text { Resistance to new } \\
\text { processes }\end{array}$ & $\begin{array}{l}\text { ITIL process champions should maintain a light-hearted approach in } \\
\text { presentations and workshops. }\end{array}$ \\
\hline $\begin{array}{l}\text { Insufficient and end- } \\
\text { user support }\end{array}$ & $\begin{array}{l}\text { Assign training personnel to each stakeholder area to provide training and } \\
\text { ongoing support after the implementation process is complete. }\end{array}$ \\
\hline $\begin{array}{l}\text { Focus on management } \\
\text { and workarounds }\end{array}$ & $\begin{array}{l}\text { Change focus to resolving underlying causes of incidents, measuring return } \\
\text { on investment. }\end{array}$ \\
\hline $\begin{array}{l}\text { Problems with } \\
\text { adaptability to change }\end{array}$ & $\begin{array}{l}\text { Assign ITIL process champions that will provide constant feedback to the } \\
\text { technical staff and provide training for IT staff. }\end{array}$ \\
\hline
\end{tabular}




\section{Issues in Information Systems}

Volume 22, Issue 2, pp. 187-198, 2021

\begin{tabular}{|l|l|}
\hline \multicolumn{2}{|c|}{ Psychological Challenges } \\
\hline Challenge/Barrier & Solution \\
\hline Resistance to change - \begin{tabular}{l} 
Loss of status/pay \\
\cline { 2 - 2 }
\end{tabular} & $\begin{array}{l}\text { Encourage stakeholders to participate in each step of the process. } \\
\text { periodically. }\end{array}$ \\
\hline Resistance to change - & Establish one-on-one communication with stakeholders. \\
\cline { 2 - 2 } Loss of comfort & Listen and respond effectively to employee concerns. \\
\hline $\begin{array}{l}\text { Lack of employee } \\
\text { support - Lack of } \\
\text { control }\end{array}$ & $\begin{array}{l}\text { Determine the most appropriate and manageable time to implement the } \\
\text { change. }\end{array}$ \\
\cline { 2 - 2 } $\begin{array}{l}\text { Lack of employee } \\
\text { support - Employee } \\
\text { confusion }\end{array}$ & Train employees in problem-overcoming techniques. \\
\cline { 2 - 2 } & $\begin{array}{l}\text { Use terms and vocabulary that are enterprise wide. } \\
\text { understanding. }\end{array}$ \\
\cline { 2 - 2 } & Write new job descriptions. \\
\cline { 2 - 2 } & $\begin{array}{l}\text { Assign someone whose main job is to facilitate communication across all } \\
\text { areas. }\end{array}$ \\
\hline Disruption of Group & Work with each person individually as well as in a group setting. \\
\cline { 2 - 2 } $\begin{array}{l}\text { Dencourage employees to broaden their interests to other areas in their } \\
\text { department or team. }\end{array}$ \\
\hline
\end{tabular}

\section{Implication of findings}

Our research has shown that while it is important to focus on managerial and technical issues during ITIL implementation, it is just as important, if not more so, to examine and address any psychological issues that may arise. Sufficient and appropriate employee involvement can make the difference between project success or failure. Often companies make the mistake of reacting impulsively to behavior that they deem unacceptable without taking the time to discover the root of the problem. A cursory investigation into this area may not be adequate to truly and thoroughly address the concerns of those involved. It is important to dig deep into any underlying factors that may influence employee behavior instead of simply reacting to perceived subversive attitudes. To ensure the best chance of success, employees must be committed to the integration. Correctly and satisfactorily addressing their concerns is the best way to earn their cooperation.

\section{Limitations and future research}

This paper leverages the psychological aspects of change and how they can be applied to a more successful ITIL adoption. There is currently a lack of research literature directly addressing concerns beyond the usual external factors such as leadership and training. Owing to this, the correlation between the activities addressing the psychological aspects of change and ultimately successful ITIL adoption is loose at best. Simply put, even though the remediations suggested in this paper are supported by research, more studies are required as to their exact affect to ITIL adoption outcomes. A more narrowly focused case study could be performed with a domestic subject to give further insight into the critical success factors, barriers, and psychology of the American employee and how they affect the success of ITIL adoption and implementation. This paper provides groundwork for future research in the area relating psychological factors to ITIL implementation which could be addressed with a domestic case study.

\section{Concluding remarks}

All successful organizations strive to gain competitive advantage in their industries. For those providing an IT service, competitive advantage can be acquired through successful ITSM. One of the most widely used 


\section{Issues in Information Systems}

Volume 22, Issue 2, pp. 187-198, 2021

frameworks for ITSM is ITIL, however, implementing ITIL best practices takes a strong commitment and resolve to overcome the challenges and barriers at all levels of the organization. While most who undertake such a process agree on the critical outward factors for success, it is vital to understand and address the psychological factors as well. When properly addressed, the psychological barriers to successful ITIL adoption become less of an obstruction which leads to a more successful ITIL implementation.

\section{References}

Berntsen, K. R. (2017). The use of ITIL and its effect on organizational culture: Bringing the employee perspective to the scene [Master's Thesis]. Østfold University College, Halden, Norway.

Chen, A. H.-C., \& Chou, S.-K. (2010). Issues in implementing information technology service management. Service Science, 1(2), 13-17.

Coch, L., \& French, J. R. P., Jr. (1948). Overcoming resistance to change. Human Relations, 1(4), 512-532.

Dent, E. B., \& Goldberg, S. G. (1999). Challenging "resistance to change." Journal of Applied Behavioral Science, 35(1), 25-41.

Eikebrokk, T. R., \& Iden, J. (2017). Strategising IT service management through ITIL implementation: Model and empirical test. Total Quality Management, 28(3), 238-265.

Esteves, R., \& Alves, P. (2013). Implementation of an information technology infrastructure library process - The resistance to change. Procedia Technology, 9, 505-510.

Flower, O. D. (1962). Overcoming resistance to change [Film]. Beverly Hills, CA: Roundtable Productions.

Folger, J. P., Poole, M. S., \& Stutman, R. K. (2013). Working through conflict: Strategies for relationships, groups, and organizations (7th ed.). Upper Saddle River, NJ: Pearson.

Iden, J., \& Eikebrokk, T. R. (2015). The impact of senior management involvement, organisational commitment and group efficacy on ITIL implementation benefits. Information Systems and eBusiness Management, 13(3), 527-552.

Kashanchi, R., \& Toland, J. (2006). Can ITIL contribute to IT/business alignment? An initial investigation. Wirtschafts Informatik, 48, 340-348.

Lawrence, P. R. (1954). How to deal with resistance to change. Harvard Business Review, 32(3), 49-57.

Malhotra, N., Zietsma, C., Morris, T., \& Smets, M. (2021). Handling resistance to change when societal and workplace logics conflict. Administrative Science Quarterly, 66(2), 475-520.

Marrone, M., \& Kolbe, L. M. (2011). Uncovering ITIL claims: IT executives' perception on benefits and business-IT alignment. Information Systems and e-Business Management, 9, 363-380.

Mohammadi, M. M., Ravasan, A. Z., \& Hamidi, H. (2015). Investigating critical success factors in implementing ITIL framework: The case of a developing country. International Journal of Standardization Research, 13(1), 74-91. 


\section{Issues in Information Systems}

Volume 22, Issue 2, pp. 187-198, 2021

Müller, S. D., \& de Lichtenberg, C. G. (2018). The culture of ITIL: Values and implementation challenges. Information Systems Management, 35(1), 49-61.

Mye, A. (2015). How can positive psychology be applied to inform and promote positive communication in the workplace? Honors Thesis]. https://digitalcommons.brockport.edu/cgi/viewcontent.cgi?article=1097\&context=honors

Neničková, H. (2011). Critical success factors for ITIL best practice usage. Economics and Management, $16,839-844$.

Obwegeser, N., Nielsen, D. T., \& Spandet, N. M. (2019). Continual process improvement for ITIL service operations: A lean perspective. Information Systems Management, 36(2), 141-167.

Pollard, C., \& Cater-Steel, A. (2009). Justifications, strategies, and critical success factors in successful ITIL implementations in U.S. and Australian companies: An exploratory study. Information Systems Management, 26(2), 164-175.

Raflesia, S. P., Surendro, K., \& Passarella, R. (2017). The user engagement impact along information technology of infrastructure library (ITIL) adoption. Proceedings of the 2017 International Conference on Electrical Engineering and Computer Science (pp. 184-187). IEEE.

What is IT service management? (n.d.). https://www.axelos.com/best-practice-solutions/itil/what-is-itservice-management

Williams, J. R. (1998). Renewable advantage: Crafting strategy through rough economic time. New York, NY: Free Press.

Yao, Z., \& Wang, X. (2010). An ITIL based ITSM practice: A case study of steel manufacturing enterprise. Proceedings of the $7^{\text {th }}$ International Conference on Service Systems and Service Management (pp. 423-427). IEEE.

Zander, A. F. (1950). Resistance to change - Its analysis and prevention. Advanced Management, 4(5), 911. 